\title{
Polymers thermophysics parameters estimation in one-dimensional case
}

\author{
K. Atchonouglo ${ }^{1,2, a}$, M. Banna ${ }^{2}$, J.-C. Dupré ${ }^{1}$, Z.-Q. Feng ${ }^{3}$ and C. Vallée ${ }^{1}$ \\ 1 L.M.S., Université de Poitiers, B Pierre et Marie Curie, 86962 Futuroscope, France \\ 2 Laboratoire sur l'Énergie Solaire, Groupe Phénomènes de Transfert et Énergétique Université de Lomé, BP 1515, Lomé, Togo \\ 3 L.M.E., Université d'Évry-Val d'Essonne, 40 rue du Pelvoux, 91020 Évry, France
}

Received 25 August 2007, accepted 28 January 2008

\begin{abstract}
This paper sets out an identification method for the thermal conductivity $\lambda$ and the heat capacity per unit volume $\rho c$ of a polymer material. The measurement of the temperature is registered with infrared camera. An inverse reading of the F.E.M. allows to perform this identification by solving a linear algebraic system coming from a least square method. The relative uncertainty obtained in experimental case are very good.
\end{abstract}

\section{Introduction}

The identification of thermal conductivity $\lambda$ and heat capacity $\rho c$ is necessary for the characterization of new materials. For example, these parameters are involved in the selection of polymers with hight heat insulation properties. Moreover, for a complete thermo-mechanical assessment, i.e. a knowledge of mechanical energy and heat source, a well accuracy on the parameters $\lambda$ and $\rho c$ is required.

Many measurement methods have been developed for the determination of thermal parameters:

- time-domain methods as impulse flash method (Degiovanni and Laurent [4], Parker et al. [12]);

- frequency-domain methods (Guimaraes et al. [7]);

- step-heating method which is a promising photothermal technique for measurement of thermal diffusivity of solids (Vozàr and Srámková [13]);

- Hot-wire and hot-stripe measurement using specially one single point transient temperature measurement, which gives access to longitudinal or/and tranversal thermal conductivities (Gobbe et al. [6], Jannot and Meukam [8], Numes dos Santos [11]).

Most of these methods needs front and rear-surface transient temperature measurement. All of them involve minimization of an objective function. However, their availability decreases when thermal conductivity and heat capacity have to be estimated simultaneously.

Only a limited amount of work is available in the literature on inverse analysis using space transient temperature measurement (Al-Khalidy [1], Ammous et al. [2], Liu and Tan [10]). Although they appear nowadays useful

\footnotetext{
${ }^{a}$ Corresponding author:

atchonouglo@lms.univ-poitiers.fr
}

because of the new possibilities of non-destructive measurement using an infrared camera. The thermal cartography of specimens surfaces made possible by infrared camera, could be used for the estimation of anisotropic and isotropic materials preperties. Particularly, in the literature, these inverse methods were developed in order to estimate time-varying initial and boundary conditions or surface conditions ill-posed (Al-Khalidy [1], Girault and Petit [5], Kurpisz and Nowak [9], Wang and Zabaras [14]).

In the present work, our aim is to develop an inverse method to estimate simultaneously thermal conductivity $\lambda$ heat capacity $\rho c$. Finite element method is used to transform the continuous partial differential equation of heat conduction into discrete differential equation, suitable for numerical computing. The least square method is used to identify the unknown parameters through minimization of a discrepancy functional. The quadratic function defined in the least square method is built from the discrete difference equation. An application of the method is developped on two materials.

\section{Direct problem}

To illustrate the methodology for developing expressions for use in simultaneously determining unknown thermal conductivity, $\lambda$ and heat capacity per unit volum, $\rho c$, in material, we consider the following transient inverse heat conduction problem. A plate of thickness $L$ is initially at the temperature $T(x, 0)=T_{0}$. For $t>0$, the boundary surface $x=0$ is subject to prescribed constant heat flux $q$, while the boundary surface $x=L$ is insulated.

We assume that the sample is homogeneous thermally insulated, infinite rectangular sample with uniform and constant thermal properties. Figure 1 shows 


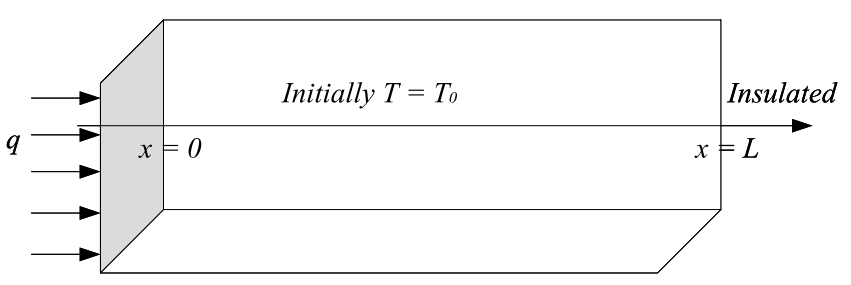

Fig. 1. Geometry and coordinates.
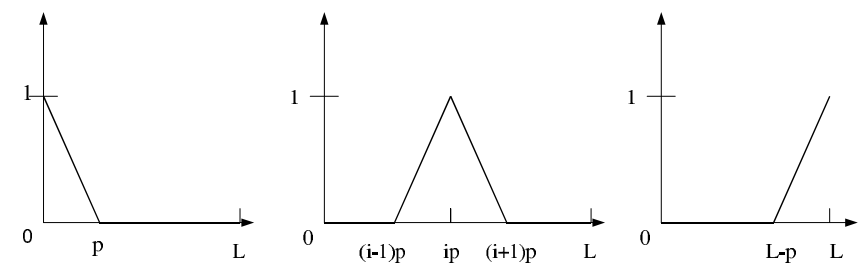

Fig. 2. Functions $\phi_{0}(x), \phi_{i}(x)$ and $\phi_{n}(x)$.

the geometry and the coordinates for the one-dimensional physical problem considered.

The formulation of the this transient heat conduction problem can be expressed as:

$$
\lambda \frac{\partial^{2} T}{\partial x^{2}}=\rho c \frac{\partial T}{\partial t}
$$

accompanied by heat flux conditions

$$
-\lambda \frac{\partial T}{\partial x}(0, t)=q(t) ; \quad \lambda \frac{\partial T}{\partial x}(L, t)=0
$$

and initial condition

$$
T(x, 0)=T_{0}(x)
$$

The direct problem considered here is concerned with the determination of the medium temperature when the thermal properties, the initial condition and the boundary conditions at $x=0$ and $x=L$ are known.

Finite element method is applied for the discretization of partial differential equation in order to solve numerically the direct problem (Bergheau and Fortunier [3]).

We choose the nodal number $n \geq 1$, the step-space $p=L / n$, and linear piecewise-continuous functions $\phi_{i}(x)$ equal to 1 at node number $i$, and zero at any other node. The graphs of these functions are exemplified in Figure 2.

The state-space approximation solution of the equation (1) is choosen as:

$$
T(x, t)=\sum_{i=0}^{n} \theta_{i}(t) \phi_{i}(x)
$$

Following the weighted residuals method, we demand that the above integrals has to be equal to zero for every shape function $\phi_{i}(x)$, i.e.,

$$
\int_{0}^{L}\left(\lambda \frac{\partial^{2} T}{\partial x^{2}}-\rho c \frac{\partial T}{\partial t}\right) \phi_{i}(x) d x-q(t) \phi_{i}(0)=0 .
$$

Inserting the approximation $(3)$ of $T(x, t)$ in $(4)$ and taking nodal temperature $\theta_{i}(t)$ out of the integrals, the following ordinary linear differential system can be obtained:

$$
\rho c C \frac{d \theta}{d t}+\lambda K \theta(t)=F(t)
$$

where $\theta=\left[\theta_{0}(t) \ldots \theta_{n}(t)\right]^{T}, C$ and $K$ are $(n+1) \times(n+1)$ symmetric matrices defined as follows:

$$
C_{i j}=\int_{0}^{L} \phi_{j}(x) \phi_{i}(x) d x \quad K_{i j}=\int_{0}^{L} \phi_{i}^{\prime}(x) \phi_{j}^{\prime}(x) d x
$$

and the vector $F(t) \in \mathbb{R}^{n+1}$ has as components $F_{i}(t)=$ $q(t) \phi_{i}(0)$. Denote that the matrix of capacity $C$ is definite positive since the conductivity matrix $K$ is positive.

The initial condition for (5) follows from (2):

$$
\theta(0)=\left[T_{0}(0) T_{0}(p) \ldots T_{0}(i p) \ldots T_{0}(L)\right]^{T} .
$$

Straightforward integrations lead to

$$
\begin{aligned}
& C_{00}=C_{n n}=2 p / 6 \quad C_{i i+1}=2 p / 6, C_{i i+1}=4 p / 6 \\
& \text { for } 1 \leq i \leq n-1 . \\
& K_{00}=K_{n n}=1 / p \quad K_{i i+1}=-1 / p \quad K_{i i}=2 / p \\
& \text { for } \quad 1 \leq i \leq n-1 .
\end{aligned}
$$

For the direct problem, equation (5) is solve numerically. The parameters $\lambda$ and $\rho c$, the step-space $p$ are chosen. Once $\lambda, \rho c$ and $p$ are fixed, there is the minimum permissible time-step size $\Delta t$ given by the following stability criterion (Bergheau and Fortunier [3], Kurpisz and Nowak [9])

$$
1 \leq \frac{6 \nu \lambda \Delta t}{\rho c p^{2}}
$$

where $\nu$ indicates the time-marching procedure to calculate $d \theta / d t$.

The temperature field is obtained by solving the direct problem with finite element method for space discretization and finite difference method for time integration.

\section{Inverse problem}

For the inverse problem, the thermal parameters $\lambda$ and $\rho c$ are regarded as being unknown. We assumed that an infrared camera is used to record the temperature information to identified $\lambda$ and $\rho c$ in the inverse calculation. Let the temperature reading taken within the infrared camera over the time period $t_{f}$ be denoted by $\theta_{i}(t), i=0$ to $n$, where $i=1$ and $n$ always correspond to $x=0$ and $L$ respectively. Then the inverse problem can be stated as follows: by utilizing the above-mentioned measured temperature data, $\theta_{i}(t)$, estimate the unknown thermal properties $\lambda$ and $\rho c$ over the time $t_{f}$. 


\subsection{Least square method}

The inverse problem can be regarded as an optimization problem which aims at finding the unknown termal property vector $\mathbf{u}=\left[\begin{array}{ll}\rho c & \lambda\end{array}\right]^{T}$ that minimizes the following quadratic function $J(.,$.$) built with the square of the dis-$ crepancy between responses of the system:

$$
\begin{aligned}
J(\mathbf{u}) & =\frac{1}{2} \int_{0}^{t_{f}}\left\|\rho c C \frac{d \theta(t)}{d t}+\lambda K \theta(t)-\mathbf{F}\right\|^{2} d t \\
& =\frac{1}{2} \mathbf{u}^{T} A \mathbf{u}-\mathbf{f}^{T} \mathbf{u}+\text { terms of degree zero }
\end{aligned}
$$

where $\|$.$\| is the norm associated to the scalar product$ $\langle.,$.$\rangle on \mathbb{R}^{n+1}$ and the matrix $A$ and vector $\mathbf{f}$ are:

$$
\begin{aligned}
& A=\int_{0}^{t_{f}}\left[\begin{array}{cc}
\left\langle C \frac{d \theta}{d t}, C \frac{d \theta}{d t}\right\rangle & \left\langle C \frac{d \theta}{d t}, K \theta\right\rangle \\
\left\langle C \frac{d \theta}{d t}, K \theta\right\rangle & \langle K \theta, K \theta\rangle
\end{array}\right] d t \\
& \mathbf{f}=\int_{0}^{t_{f}}\left[\begin{array}{c}
\left\langle C \frac{d \theta}{d t}, \mathbf{F}\right\rangle \\
\langle K \theta, \mathbf{F}\rangle
\end{array}\right] d t .
\end{aligned}
$$

The minimum of the quadratic function $J$ is attained when the gradient of the functional $J(\mathbf{u})$ is equal to zero, i.e.

$$
A \mathbf{u}=\mathbf{f}
$$

\subsection{Existence and uniqueness of solution}

We denote by $A$ the above $2 \times 2$ symmetric matrix and by $f$ the vector of the second member. To identify the

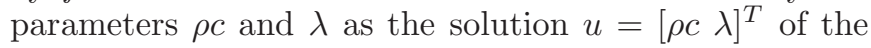
equation $A \mathbf{u}=f$, the matrix $A$ has to be invertible. This property is ensured by its definite positiveness.

Indeed, for $V \in \mathbb{R}^{2},\langle A V, V\rangle=\int_{0}^{t_{f}} \| V_{1} C \frac{d \theta}{d t}+$ $V_{2} K \theta \|^{2} d t \geq 0$. Then the matrix $A$ is positive.

For $V \in \mathbb{R}^{2}\langle A V, V\rangle=0$ can happen only if $\forall t \in$ $\left[0, t_{f}\right], V_{1} C d \theta / d t+V_{2} K \theta=0$.

As the vector $\theta(t)$ is solution of $(5)$, then $V_{1}=V_{2}=0$. This proves that the matrix $A$ is definite positive; then the parameters $\lambda$ and $\rho c$ can be estimated simultaneously.

\subsection{Algorithm}

For the solving of the inverse problem, the calculations are carried out in the following manner;

(1) compute the 3 coefficients of the matrix $A$ by numerical integration;

(2) compute the 2 coefficients of the vector $\mathbf{f}$ by numerical integration;

(3) solve algebraically the equation $A \mathbf{u}=\mathbf{f}$.

These tree steps constitute the solution algorithm for the above inverse problem.

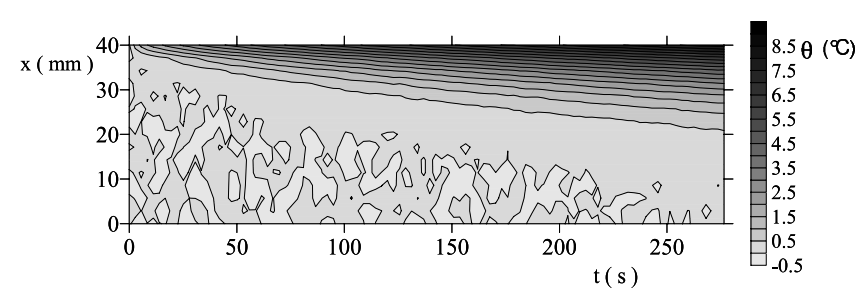

Fig. 3. Thermal space-time cartography.

\section{Algorithm validation}

\subsection{Identification algorithm test using numerical simulation transient temperature data}

To demonstrate the validity and the accuracy of the method of inverse analysis considered for simultaneous estimation of thermal conductivity and heat capacity per unit volum, we present the following simulation.

We choose $\lambda_{\text {sim }}=0.45 \mathrm{~W} \cdot \mathrm{m}^{-1}{ }^{\circ} \mathrm{C}^{-1}$ and $\rho c_{\text {sim }}=$ $1.74 \mathrm{~J} \cdot \mathrm{mm}^{-3} \cdot{ }^{\circ} \mathrm{C}^{-1}$ for polyethylen.

The lenght of the sample is $L=40 \mathrm{~mm}$. The time increments is $\Delta t=3.5 \mathrm{~s}$ and the space steps $p=L / n$.

Initial temperature of the medium is $T_{0}=20^{\circ} \mathrm{C}$ and the applied constant heat flux is $q=427.345 \mathrm{~W} \cdot \mathrm{m}^{-2}$.

To simulate the measured temperatures that contain measurement errors, random error $\omega \sigma$ are introduced to the exact temperatures as

$$
\theta_{i}=\Theta_{i}+\omega \sigma
$$

where $\Theta_{i}$ is determined from the solution of the direct problem $(5,6)$. A random-number generator is used to generate values for $\omega(-1 \leq \omega \leq+1), \sigma=0.02{ }^{\circ} \mathrm{C}$ is a standard deviation choosen for the measurement errors as given by the performances of the "actual" infrared camera; a systematic error $F_{s}=0.05, F(t)$ is added to the heat vector $F(t)$.

Figure 3 shows a thermal cartography.

\subsection{Results analysis}

The simulated field of temperature is used to illustrate the validity and accuracy of the algorithm.

Figures 4 and 5 demonstrate the effect of the time control of heat flux and the nodal number $n$. The graphs of identified thermal properties (thermal conductivity $\lambda_{\text {id }}$ and heat capacity per unit volum $\rho c_{\mathrm{id}}$ ) and simulated values are given Figure 4.

We are limited by the criterion (7) and we cannot choose the space step less than a pixel. The analysis shows that the thermal properties $\lambda$ and $\rho c$ can be estimated simultaneously for $t_{f}=1 s$ and $p \approx 0.66 \mathrm{~mm}$.

The results show that in the case of solving parabolic inverse problem with noise in data, an excellent agreement with the simulated values is obtained both for thermal conductivity $\lambda$ and the heat capacity per unit volum $\rho c$.

It results from precision analysis that the better agreement bettween the simulated and the estimated values is 




Fig. 4. Profile of $\rho c_{\mathrm{id}}-\rho c_{\mathrm{sim}}\left(\mathrm{J} \cdot \mathrm{m}^{-3} \cdot{ }^{\circ} \mathrm{C}^{-1}\right)$.

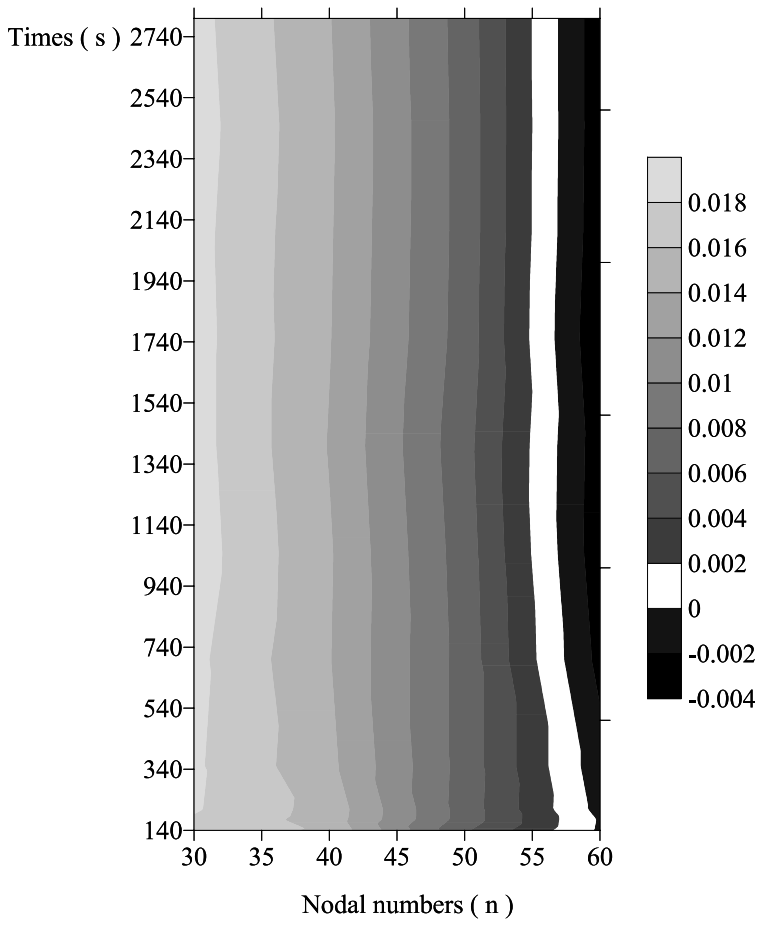

Fig. 5. Profile of $\lambda_{\mathrm{id}}-\lambda_{\mathrm{sim}}\left(\mathrm{W} \cdot \mathrm{m}^{-1} \cdot{ }^{\circ} \mathrm{C}^{-1}\right)$.

obtained for $n=60$. The time-step was choosen in idea to have the step-space more or less to a pixel in idea to be in experimental means, especially to the recording of the infrared camera. Table 1 shows that for the simulated data with no error (i.e. $\sigma=0{ }^{\circ} \mathrm{C}$ ), the solution produces exact results for the computed thermal properties. For
Table 1. Simulation Results.

\begin{tabular}{lll}
\hline & $\sigma=0{ }^{\circ} \mathrm{C}$ & $\sigma=0.02{ }^{\circ} \mathrm{C}$ \\
\hline$\lambda\left(\mathrm{W} \cdot \mathrm{m}^{-1} \cdot{ }^{\circ} \mathrm{C}^{-1}\right)$ & 0.4495 & 0.4497 \\
$\rho c\left(\mathrm{~J} \cdot \mathrm{m}^{-3} \cdot{ }^{\circ} \mathrm{C}^{-1}\right)$ & $1.75 \times 10^{6}$ & $1.7889 \times 10^{6}$ \\
\hline
\end{tabular}

Table 2. Manufacturers values.

\begin{tabular}{lcc}
\hline & $\lambda\left(\mathrm{W} \cdot \mathrm{m}^{-1} \cdot{ }^{\circ} \mathrm{C}\right)$ & $\rho c\left(\mathrm{~J} \cdot \mathrm{m}^{-3} \cdot{ }^{\circ} \mathrm{C}^{-1}\right)$ \\
\hline PET & $0.42-0.51$ & $1.786 \times 10^{6}$ \\
PMMA & $0.17-0.19$ & $1.66 \times 10^{6}-1.785 \times 10^{6}$ \\
\hline
\end{tabular}

$\sigma=0.02{ }^{\circ} \mathrm{C}$, the relative uncertainties on thermal properties $\lambda$ and $\rho c$ are respectively $0.06 \%$ and $2.8 \%$.

\section{Identification algorithm test using experimental measurements data}

\subsection{Experimental manipulation}

The present experimental facility featured an heating thread sandwiched between two identical test pieces. The two plates are identical in terms of composition, density, thermal properties, dimensions, thermal boundary condition, etc.; therefore, identical thermal behavior was expected. In such an arrangement, each specimen was subjected to a heat input rate equal to half of the total heat rate generated by the heater. All the other surfaces of the test-tube except the lateral ones were insulated in order to eliminate heat loss, if any. An infrared camera was used to tape the temperature distribution throughout specimen during heating. A flexible hot strip was sandwiched between the two test-tubes, and its heating power was controlled by an electric generator, with the power measured by a voltmeter and an ammeter. A video monitor allowed the visualization and the control of the one-dimensional heat flows along the plates.

The experimental measure carried on two polumeys: polyethylen or PET and plexiglass or PMMA. The manufacturers values are given in Table 2 .

The lenght each plate is $L=40 \mathrm{~mm}$; the step-space $p=L / 60$ and the time increment is $\Delta t \approx 3.5 \mathrm{~s}$.

The materials has initial temperature $T_{0}=20{ }^{\circ} \mathrm{C}$, when $t>0$, and the boundary surface $x=0$ is subjected to a constant heat flux, $q=427.345 \mathrm{~W} \cdot \mathrm{m}^{-2}$ (the heat flux is the power of the generator).

Figure 6 shows the experimental apparatus and the thermal cartography of polyethyelen in given is Figure 7.

\subsection{Results analysis}

The solution algorithm presented is this paper is used to identified the thermal properties $\lambda$ and $\rho c$ of the two polymers.

Compared to literature values of thermal parameters for polyethylen and plexiglass, the experimental results are obtained with good precisions. 


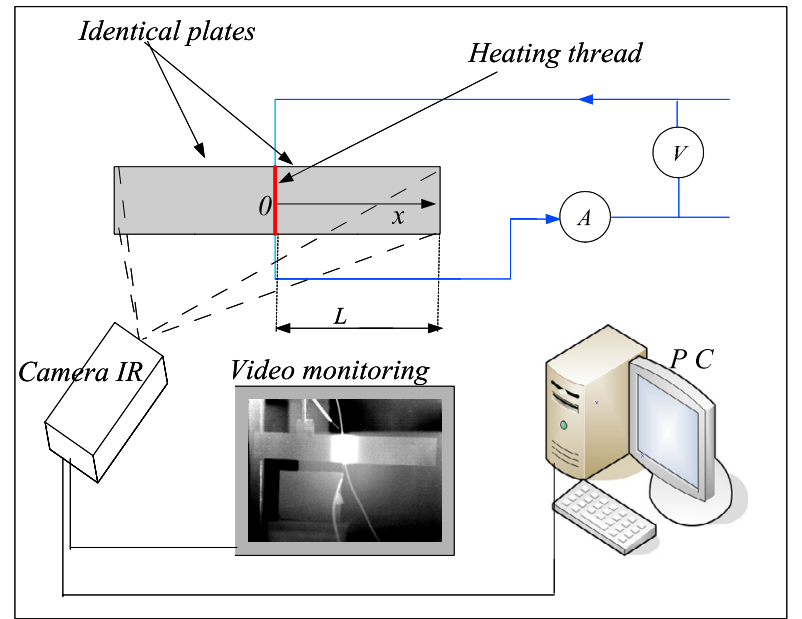

Fig. 6. Heater-sample assembly in the specimen.



Fig. 7. Thermal cartography $T^{\circ} \mathrm{C}$.

Table 3. Experimental results.

\begin{tabular}{lcc}
\hline & $\lambda\left(\mathrm{W} \cdot \mathrm{m}^{-1} \cdot{ }^{\circ} \mathrm{C}^{-1}\right)$ & $\rho c\left(\mathrm{~J} \cdot \mathrm{m}^{-3} \cdot{ }^{\circ} \mathrm{C}^{-1}\right)$ \\
\hline PET & $0.431 \pm 0.015$ & $(1.783 \pm 0.028) \times 10^{6}$ \\
PMMA & $0.185 \pm 0.009$ & $(1.734 \pm 0.03) \times 10^{6}$ \\
\hline
\end{tabular}

Since the variation in temperature is small and the ambiant temperature $T_{a}=20^{\circ} \mathrm{C}$, these values of thermal parameters $\lambda$ and $\rho c$ can be regarded us termal parameters at $T=20{ }^{\circ} \mathrm{C}$.

The maximum deviations of the experimental points are $2 \%$, while the relative uncertainty on the parameters $\lambda$ and $\rho c$ are respectively $3.5 \%$ and $2.13 \%$ for polyethylen, $6.3 \%$ and $2.24 \%$ for plexiglass.

\section{Conclusion}

The associated direct problem was solved with a finite element discretization technique. Determinant analysis of the information matrix shows that, it is possible to estimate simultaneously thermal conductivity and heat capacity by using knowing temperature data. There is a good agreement between the exact and estimated properties. The development and the interest of the inverse method described in this work provide a reliable procedure in the estimation of the thermal properties of the multidimensional and the non linear inverse heat conduction problems.

\section{References}

1. N. Al-Khalidy. Int. J. Heat Mass Transfer 44, 3731 (1998)

2. A. Ammous, F. Sellami, K. Ammous, H. Morel. Int. J. Thermal Sci. 42, 533, (2003)

3. J.-M., Bergheau, R. Fortunier, in Simulation numerique des transferts thermiques par elements finis (Lavoisier, 2004)

4. A. Degiovanni, M. Laurent. Revue de Physique Appliquée 21, 229 (1986)

5. M. Girault, D. Petit. Int. J. Heat Mass Transfer 48, 119 (2005)

6. C. Gobbe, S. Iserna, B. Ladevie. Int. J. Thermal Sci. 43 951 (2004)

7. G. Guimaraes, P.C. Philippi, P. Thery. Review of Scientific Instruments 66 (1996)

8. Y. Jannot, P. Meukam. Publishing. Mesurement Science and Technology 15, 1932 (2004)

9. K. Kurpisz, A.J. Nowak, in Inverse Thermal Problems (Technical University of Silesea, Poland, 1995)

10. L.H. Liu, H.P. Tan. Journal of Quantitative Spectroscopy and Radiative Transfer 83, 333 (2004)

11. W. Numes dos Santos. Polymer Testing, 24932 (2005)

12. W.J. Parker, R.J. Jenkins, C.P. Butter, G.L. Abbot. Flash J. Appl. Phys. 32, 1679 (1961)

13. L. Vozàr, T. Srámková. Int. J. Heat Mass Transfer 40, 1647 (1997)

14. J. Wang, N. Zabaras. Int. J. Heat Mass Transfer 48, 15 (2005) 